\title{
RESISTÊNCIA DO SOLO À PENETRAÇÃO EM RESPOSTA AO NÚMERO DE AMOSTRAS E TIPO DE AMOSTRAGEM(1)
}

\author{
João Tavares Filho ${ }^{(2)}$ \& Adriana Aparecida Ribon ${ }^{(3)}$
}

\begin{abstract}
RESUMO
O monitoramento periódico do estado de compactação do solo por meio da resistência à penetração é uma forma prática de avaliação dos efeitos dos diferentes sistemas de manejo na estrutura do solo e do crescimento radicular das culturas. Este trabalho teve como objetivo avaliar a variação dos valores de resistência do solo à penetração em resposta ao número de repetições (população amostral) a partir de diferentes formas de amostragem em campo, em um Latossolo Vermelho eutroférrico, sob três tipos de manejo: plantio direto (PD), cultura perene (CP) e preparo convencional (PC). $\mathrm{O}$ trabalho foi realizado em uma área situada na região norte do Estado do Paraná. Nessa área, foram feitas amostragens em três subáreas de 1 ha para determinação da resistência do solo à penetração por meio de um penetrômetro de Impacto modelo IAA/Planalsucar/Stolf, nas profundidades de $0-0,10 ; 0,10-0,20 ; 0,20-0,40 ; 0,40-0,60 \mathrm{~m}$. Foram utilizados dois esquemas de amostragens na área: uma feita de forma sistemática (rede de pontos distantes de $25 \mathrm{~m}$ entre si) e outra totalmente ao acaso com $1,3,5,10,15,20,30,40$ e 50 repetições. Para todos os pontos e profundidades, foram determinados o valor médio da resistência à penetração (MPa), o intervalo de confiança e a exatidão da estimativa (D) das médias de penetrometria por meio da teoria estatística clássica, levando em consideração o número de amostras (n) e o desvio-padrão da amostra (S). O nível de significância utilizado foi de 0,05 . Nas condições em que foi realizado o trabalho, os resultados obtidos permitiram concluir que o número de amostras representativas para a determinação da resistência do solo à penetração não variou de forma significativa com o tipo de amostragem. Porém, os intervalos de confiança foram sempre menores na amostragem sistemática. O número de amostras representativas para a determinação da resistência do solo à penetração variou com o sistema de manejo e a profundidade de amostragem. A melhor
\end{abstract}

\footnotetext{
(1) Recebido para publicação em julho de 2007 e aprovado em novembro de 2007.

(2) Professor do Departamento de Agronomia da Universidade Estadual de Londrina - UEL. Caixa Postal 6001, CEP 86051-990 Londrina (PR). E-mail: tavares@uel.br

(3) Professora da Universidade Católica Dom Bosco - UCDB. Fazenda Escola São Vicente. Avenida Tamandaré 6000, Jardim Seminário, Caixa Postal 100, CEP 79117-900 Campo Grande (MS). E:mail: aaribon@yahoo.com.br.
} 
representatividade da média dos resultados de resistência do solo à penetração ocorreu para $n \geq 15$ (PD e CP) ou $n \geq 20(0-0,10 \mathrm{~m})$ e $15(0,20-0,60 \mathrm{~m})$ no caso do PC. A população amostral $n \geq 10$ na profundidade $0-0,60 \mathrm{~m}$, nos dois tipos de amostragem e manejos de solo, permitiu alta exatidão dos dados, tornando os parâmetros estatísticos mais confiáveis, com homogeneidade nos resultados e linearidade apresentada nas curvas das populações amostrais a partir do erro amostral de $10 \%$

Termos de indexação: amostragem de solo, manejo de solo, compactação de solo.

\title{
SUMMARY: VARIATION IN SOIL PENETRATION RESISTANCE IN RESPONSE TO SAMPLE NUMBER AND SAMPLING TYPE
}

\begin{abstract}
Monitoring the state of soil compaction periodically by assessing soil penetration resistance is a practical way of evaluating the effects of different management systems on the soil structure and crop root development. This study aimed to evaluate the variation of soil penetration resistance in response to the number of replications (sample population) of different field sampling forms of an Oxisol under three management types: non-tillage $(P D)$, perennial crop $(C P)$ and conventional tillage $(P C)$. The experiment was carried out in the Northern Paraná State, Brazil. Samples were collected in three sub-areas of 1 ha to determine soil penetration resistance at different depths $(0-0.10 ; 0.10-0.20 ; 0.20-0.40$; $0.40-0.60 \mathrm{~m}$ ). Sampling was carried out as follows: systematic sampling (grid points, spaced $25 \mathrm{~m}$ apart) and completely randomized sampling, with 1, 3, 5, 10, 15, 20, 30, 40, and 50 replications. For all points and depths it was determined the average value of penetration resistance $(\mathrm{MPa})$, the confidence interval and estimation accuracy $(D)$ of the penetrometer measurements through classical statistical theory based on the number of samples $(n)$ and standard deviation of the sample (S), at a significance level of 0.05 . For the given experimental conditions (sub-areas of 1 ha Oxisol under three different managements), results indicated that the number of representative samples to determine soil penetration resistance did not vary significantly with the sampling type. However, the confidence intervals were always lower when it was adopted systematic sampling and the number of representative samples to determine soil penetration resistance varied with management system and sampling depth. The mean soil penetration resistance was more representative for $n \geq 15$ (DP and CP) or $n \geq 20(0-0.10 \mathrm{~m})$ and $15(0.20-0.60 \mathrm{~m})$ for PC; the population sample $n 10$ in the $0-0.60$ m layer by both sampling types and soil managements studied enabled the obtaining a high accuracy of the data and the statistical parameters more reliable, with a certain homogeneity and linearity of the results presented in curves of the sample populations, considering a sample error of $10 \%$.
\end{abstract}

Index terms: management, sampling, soil penetration resistance, soil compaction.

\section{INTRODUÇÃO}

O manejo do solo tem a finalidade de criar condições estruturais favoráveis ao crescimento e desenvolvimento das culturas (parte aérea e raízes). O penetrômetro de impacto para determinação da resistência do solo à penetração (Stolf et al., 1983; Stolf, 1991; Camargo \& Alleoni, 1997), associado ao estudo do perfil cultural, (Tavares-Filho et al., 1999a,b) tem sido usado para correlacionar e explicar os efeitos da compactação no crescimento radicular e, por conseqüência, das culturas. Segundo Tavares-Filho et al. (2001), um diagnóstico qualitativo (distribuição espa- cial das estruturas no perfil do solo) e quantitativo (grau da compactação do solo) ganha importância para auxiliar na verificação da qualidade do manejo utilizado e também no estabelecimento de limites de compactação que não afetem o crescimento radicular das plantas nos diferentes sistemas de manejo.

Dessa forma, o monitoramento periódico do estado de compactação do solo por meio da resistência à penetração é uma forma prática de avaliação dos efeitos dos diferentes sistemas de manejo na estrutura do solo e no crescimento radicular das diferentes culturas, permitindo assim pesquisas e avaliações na propriedade rural. Somente com acompanhamento 
ou avaliação periódica dessa resistência do solo à penetração é que o agricultor poderá conhecer, de forma mais detalhada, os efeitos provocados pelos diferentes sistemas de manejo e coletar subsídios importantes para sua conservação (Camargo \& Alleoni, 1997) e melhoria de produtividade.

Assim, a determinação de um número de amostras que assegure a obtenção de um valor médio confiável da área avaliada permitirá economia de tempo e trabalho e a realização sistemática das amostragens necessárias para o acompanhamento do sistema de produção, uma vez que haverá uma relação custobenefício positiva por meio da acurária na avaliação com menor custo. Para o pesquisador, a intensidade de amostragem ideal para os vários ambientes, usos e manejos deve ser alta para aumentar a probabilidade de estabelecer corretamente as relações de causa e efeito (Clay et al., 1999). No caso de trabalho em propriedade rural, uma amostragem menos intensiva, com menor custo, talvez seja mais apropriada.

Pesquisas que informam um número de amostras representativas para estudos com penetrômetros de impacto são escassas e as repetições feitas em campo são variáveis, segundo a relação custo-benefício que cada um acha mais propícia, o que pode levar a repetições de campo não suficientes para avaliação dessa propriedade e a resultados não representativos da área considerada. Esse fato pode, por exemplo, levar a decisões quanto ao preparo do solo com base em uma interpretação errônea do seu estado de compactação devido à variabilidade dessa propriedade física. Para Torres \& Saraiva (1999), as leituras com penetrômetros são importantes para a definição das áreas com mais problemas de compactação, sendo, para isso, necessária uma amostragem intensa.

Basicamente, em solos são usados dois tipos de amostragens: ao acaso ou clássica e a sistemática. Amostragem ao acaso ou clássica é aquela em que as amostras são retiradas de uma população sem seguir um critério definido. A relação entre o volume de amostras do solo e a variabilidade das propriedades nas amostras pode ser definida pelo conceito de volume elementar representativo. $\mathrm{O}$ tamanho das amostras indica o volume elementar representativo da média geral da propriedade a ser estudada. Bear (1972) apresentou uma definição prática de volume elementar representativo: é o volume de solo que contém uma representação máxima de variações microscópicas em toda a forma e proporções do sistema. Algumas pesquisas vêm utilizando esse conceito em diversos tipos de estudos com propriedades físicas do solo (Lauren et al., 1988; Buchter et al., 1994; Mallants et al., 1997; Ribon \& Tavares Filho, 2002).

$\mathrm{Na}$ amostragem sistemática é estabelecido um plano criterioso, ou seja, a coleta é feita seguindo-se uma rede de pontos, com distâncias predefinidas, oferecendo assim maior representatividade da área. Para Petersen \& Calvin (1965), a amostragem sistemática é mais rigorosa já que não há a influência do amostrador, mas há melhor cobertura da área amostrada, o que permite melhor representatividade.

Assim, este trabalho teve como objetivo verificar a variação dos valores de resistência do solo à penetração em resposta ao número de repetições (população amostral) a partir de diferentes formas de amostragem em campo, em um Latossolo Vermelho eutroférrico, sob três tipos de manejo: plantio direto (PD), cultura perene $(\mathrm{CP})$ e preparo convencional (PC).

\section{MATERIAL E MÉTODOS}

O trabalho foi realizado em área de Latossolo Vermelho eutroférrico (teor de argila entre 790 e $800 \mathrm{~g} \mathrm{~kg}^{-1}$ ), sob três manejos diferentes: 14 anos no sistema de plantio direto (PD) (produção de soja, milho, trigo, aveia), preparo convencional (PC) (produção de soja, trigo, aveia), aração (arado de disco) e gradagem superficiais $(0,10-0,15 \mathrm{~m})$ e cultura perene $(\mathrm{CP})$ (citrus implantado a 20 anos), situada na região norte do Estado do Paraná, com características climáticas Cfasubtropical úmido, segundo classificação de Köpen. Nessa área, selecionaram-se três subáreas de 1 ha, próximas entre si, com declive variando de $4 \%$ (área sob plantio direto e preparo convencional) a $6 \%$ (área sob cultura perene), onde foram feitas amostragens para determinação da resistência do solo à penetração por meio de um penetrômetro de Impacto modelo IAA/ Planalsucar/Stolf, para as profundidades de $0-0,10$; $0,10-0,20 ; 0,20-0,40 ; 0,40-0,60 \mathrm{~m}$ e para cada profundidade. $\mathrm{O}$ número de impactos $\mathrm{dm}^{-1}$ foi transformado em resistência dinâmica (MPa) por meio da equação proposta por Stolf (1991), ou seja, $\mathrm{RP}\left(\mathrm{kgf} \mathrm{cm}^{-2}\right)=5,6+6,89 \mathrm{~N}\left(\right.$ impactos dm $\left.{ }^{-1}\right)$ e, para converter a RP em kgf cm${ }^{-2}$ para MPa, multiplicouse o resultado obtido pela constante 0,098 .

Foram utilizados dois esquemas de amostragens nas referidas áreas: uma feita de forma sistemática e outra de forma totalmente causualizada com $1,3,5$, 10, 15, 20, 30, 40 e 50 repetições. Esses pontos levantados em campo foram analisados a partir do conceito de que, aumentando o número de repetições por ha, menor é a variabilidade de um valor representativo da propriedade estudada em escala maior.

Para a amostragem sistemática, o sistema de coletas foi uma adaptação do esquema de amostragem sistemática de Petersen \& Calvin (1965), no qual a coleta é feita seguindo-se uma rede de pontos, oferecendo assim maior representatividade da área. As três áreas avaliadas foram demarcadas com quadrados de $25 \mathrm{~m}$ de lado, por acreditar que a distância de $25 \mathrm{~m}$ garante a independência das observações. Nos vértices dos quadrados, foram realizadas as amostragens com o penetrômetro de impacto. O número de repetições foi igual ao número de pontos ( 25 pontos) para cada área. Para todas as 
profundidades, foi determinado o número ideal de amostras por meio da fórmula proposta por Petersen $\&$ Calvin (1965): $n=\frac{t_{\alpha}{ }^{2} \times S^{2}}{D^{2}}$, em que $\mathrm{n}=$ número de amostras; $\mathrm{t}=$ valor da tabela de distribuição " $\mathrm{t}$ " em função do nível de significância $(\alpha)$ e do número de graus de liberdade com que se estimou a variância amostral; $\mathrm{S}=$ desvio-padrão da amostra; $\mathrm{D}=$ produto da média $(\mathrm{m})$ pela percentagem de variação em torno da média. Foram considerados $10 \%$ de variação em torno da média e coeficiente de confiança $(1-\alpha)$ de $99 \%$. Além disso, para todos os pontos e profundidades, foram determinados os valores médios de resistência à penetração, a intervalo de confiança e a precisão da estimativa (D) das médias de penetrometria por meio da teoria estatística clássica, levando em consideração o número de amostras (n) e o desvio-padrão da amostra (S). O nível de significância utilizado foi de 0,05.

$\mathrm{O}$ trabalho de penetrometria em campo foi realizado em janeiro de 2007 e, no momento de utilização desse instrumento, foi determinada a umidade gravimétrica (Quadro 1) para as diferentes profundidades, a partir de amostras coletadas ao acaso com trado holandês, num total de 10 pontos em cada subárea estudada.

\section{RESULTADOS E DISCUSSÃO}

Verificou-se, no caso do plantio direto (PD) e da cultura perene (CP) (Figuras 1 e 2), que os valores

\section{Quadro 1. Umidade do Latossolo Vermelho sob três diferentes manejos, determinada no momento de realização da penetrômetria de impacto}

\begin{tabular}{|c|c|c|}
\hline Subárea & Profundidade & Umidade \\
\hline \multirow{5}{*}{ Plantio direto } & $\mathrm{m}$ & $\mathrm{kg} \mathrm{kg}^{-1}$ \\
\hline & $0-0,10$ & $0,19 \pm 0,02$ \\
\hline & $0,10-0,20$ & $0,22 \pm 0,03$ \\
\hline & $0,20-0,40$ & $0,24 \pm 0,04$ \\
\hline & $0,40-0,60$ & $0,23 \pm 0,03$ \\
\hline \multirow[t]{4}{*}{ Cultura perene } & $0-0,10$ & $0,18 \pm 0,01$ \\
\hline & $0,10-0,20$ & $0,20 \pm 0,04$ \\
\hline & $0,20-0,40$ & $0,21 \pm 0,01$ \\
\hline & $0,40-0,60$ & $0,20 \pm 0,02$ \\
\hline \multirow[t]{4}{*}{ Preparo convencional } & $0-0,10$ & $0,16 \pm 0,03$ \\
\hline & $0,10-0,20$ & $0,18 \pm 0,05$ \\
\hline & $0,20-0,40$ & $0,20 \pm 0,03$ \\
\hline & $0,40-0,60$ & $0,21 \pm 0,04$ \\
\hline
\end{tabular}

médios e os intervalos de confiança da resistência do solo à penetração apresentaram semelhanças, pois o manejo foi mínimo e os valores foram influenciados pela população amostral em todas as profundidades analisadas, sem, no entanto, haver diferença significativa, a $5 \%$, entre as médias da resistência das diferentes populações amostrais. A partir de $n=3$ até $\mathrm{n}<15$, houve elevada variabilidade nos resultados de resistências encontrados nos dois casos. Porém, para $\mathrm{n} \geq 15$ (em todas as profundidades), houve estacionaridade dos dados nos dois manejos, o que se pode verificar pelo valor médio da resistência, que se repete a partir desse ponto, apresentando tendência linear, não diferindo entre as diferentes populações amostrais. Isso significa que, nas condições do trabalho, houve relação entre o tamanho de amostra $(n \geq 15)$ e o valor médio representativo de resistência do solo à penetração. Esses valores ficaram próximos àqueles encontrados para a média da resistência do solo a partir da amostragem sistemática (Figuras $1 \mathrm{e}$ 2), ou seja: para a profundidade de $0-0,10 \mathrm{~m} \mathrm{n}=18(\mathrm{PD}$ e CP); para a profundidade de $0,10-0,20 \mathrm{~m}, \mathrm{n}=18(\mathrm{PD})$ e 17(CP); na de 0,20-0,40 m, n = 16 (PD) e 15(CP); e na de $0,40-0,60 \mathrm{~m}, \mathrm{n}=13(\mathrm{PD})$ e $14(\mathrm{CP})$.

Na figura 3 são apresentados os valores médios de resistência do Latossolo Vermelho eutroférrico à penetração sob preparo convencional e seus respectivos intervalos de confiança, em função da amostragem ao acaso e da sistemática (rede de pontos distantes de $25 \mathrm{~m}$ entre si) nas diferentes profundidades avaliadas. Verifica-se que esses valores foram bem mais influenciados pela população amostral do que naqueles da amostra sob plantio direto, em todas profundidades analisadas, porém sem diferença significativa, a $5 \%$, entre as médias da resistência das diferentes populações amostrais. A partir de $\mathrm{n}=3$ até $\mathrm{n}<20$, houve alta variabilidade nos resultados de resistência. Porém, para $\mathrm{n} \geq 20$ (todas as profundidades), houve estacionaridade dos dados, o que se pode verificar na repetição do valor médio da resistência a partir desse ponto, apresentando tendência linear (não difere entre as diferentes populações amostrais). Nas condições do trabalho, houve, então, uma relação entre esse tamanho de amostra e o valor médio representativo de resistência do solo à penetração. Esses valores foram próximos àqueles encontrados para a média da resistência do solo a partir da amostragem sistemática (Figura 3), ou seja, na profundidade de $0-0,10 \mathrm{~m}$, $\mathrm{n}=22$; na de $0,10-0,20 \mathrm{~m}, \mathrm{n}=21$; na de $0,20-0,40 \mathrm{~m}$, $\mathrm{n}=18$; e na de $0,40-0,60 \mathrm{~m}, \mathrm{n}=16$.

De maneira generalizada, com os dois tipos de amostragem e os manejos $\mathrm{PD}$ e CP, verificou-se que o número de amostras para a determinação da resistência do solo, em todas as profundidades estudadas (0-0,60 m), ficou em torno de $\mathrm{n} \geq 15$; para o PC, em torno de $\mathrm{n} \geq 20$ na camada de $(0-0,10 \mathrm{~m})$; e para as camadas mais profundas do solo $(0,20-0,60 \mathrm{~m})$, em torno de $n \geq 15$ amostras, o que pode estar refletindo o fato desse manejo de solo provocar mudanças constantes na estrutura da superfície do solo, pelo uso 


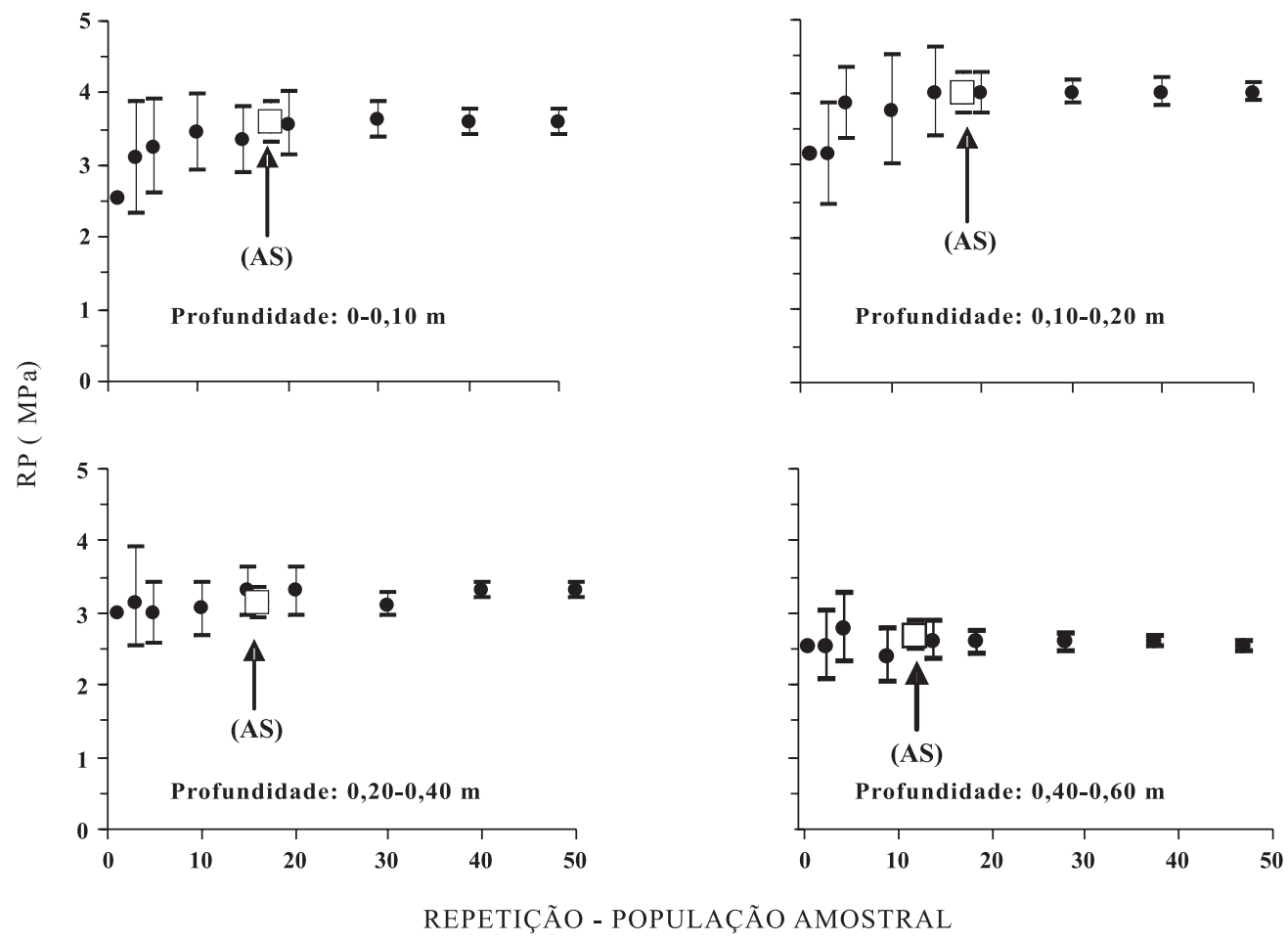

Figura 1. Valores médios e intervalo de confiança (95 \%) da resistência do solo à penetração (RP) função da amostragem ao acaso (clássica) e da amostragem sistemática (AS), com penetrômetro de impacto em diferentes profundidades de um Latossolo Vermelho eutroférrico sob plantio direto.

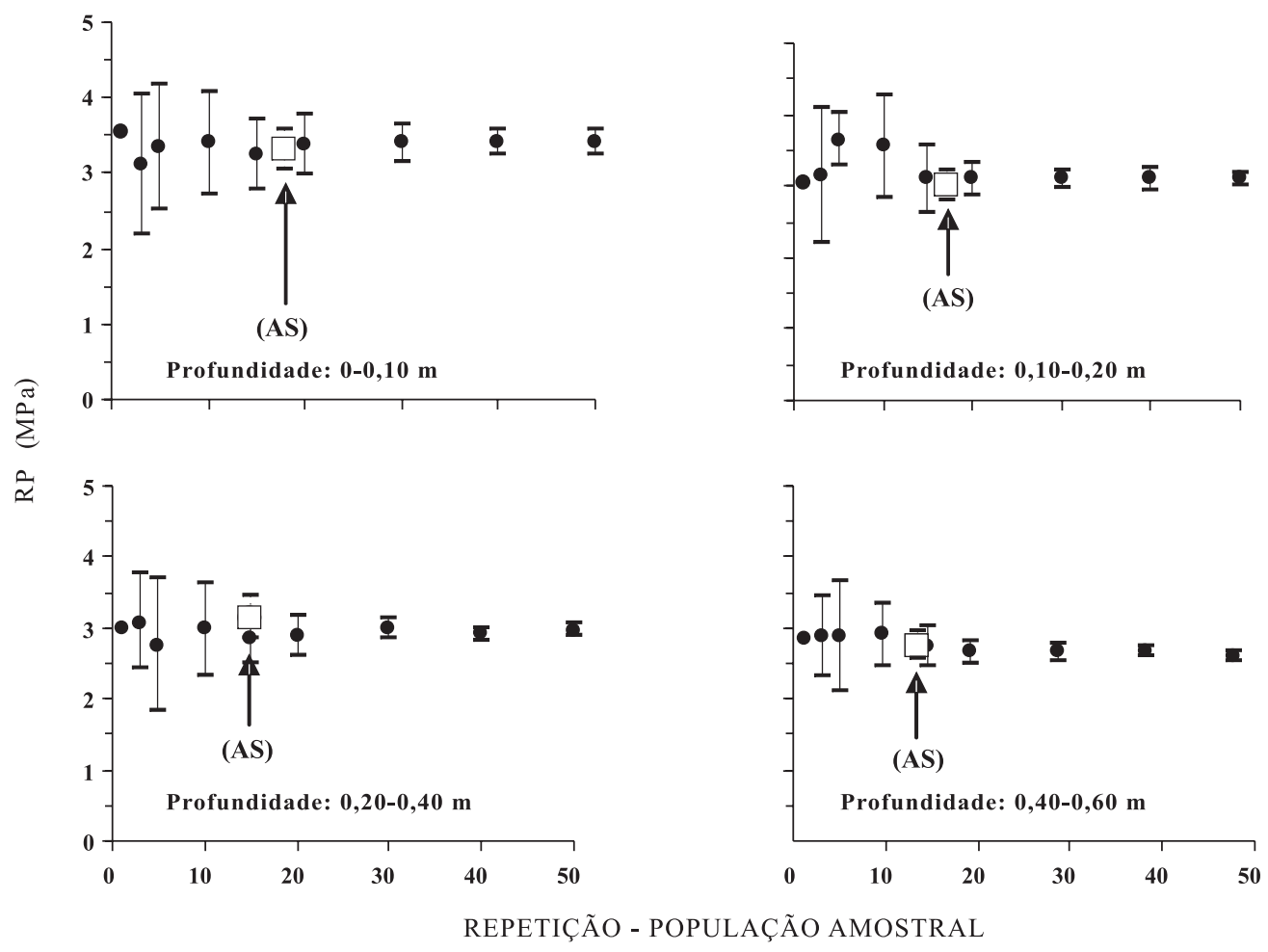

Figura 2. Valores médios e intervalo de confiança (95 \%) da resistência do solo à penetração (RP) função da amostragem ao acaso (clássica) e da amostragem sistemática (AS), com penetrômetro de impacto em diferentes profundidades de um Latossolo Vermelho eutroférrico sob cultura perene. 
constante de aração e gradagem. Nas camadas mais profundas, esse fato pode significar maior variabilidade dos resultados, exigindo maior número de amostras para a obtenção de média mais confiável nesse caso.

É comum encontrar elevados coeficientes de variação para a resistência do solo à penetração (Tormena et al., 1998; Imhoff et al., 2000; Ribon \& Tavares Filho, 2002, 2004). Silva et al. (1989) explicaram a variabilidade espacial desta propriedade física encontrada em um Latossolo Vermelho-Escuro, em amostras coletadas ao longo de uma transeção, relacionando-a ao efeito de diferentes tipos de máquinas e implementos utilizados na área. Portanto, a partir dos resultados encontrados, pode-se considerar que quanto mais o número de repetições com penetrômetro de impacto se aproximar de $\mathrm{n}=15$ (PD e CP) ou $n=20(0-0,10 \mathrm{~m})$ e $\mathrm{n}=15(0,20-0,60 \mathrm{~m})$ no caso do PC, melhor será a representatividade da média dos resultados, pois, a partir deste número de amostras, não haverá nenhuma melhoria significativa na confiabilidade das médias (nenhum ganho em acurácia na estimativa da média) de resistência do solo à penetração nas condições deste trabalho. Esses resultados estão de acordo com os valores encontrados na literatura (Jobim, 1996; Torres \& Saraiva, 1999; Embrapa, 2002), que indicam um número mínimo de amostras em torno de 10-15 subamostras por gleba amostrada, porém, não especificam o método utilizado para sua definição. Esses números diferem dos encontrados por Ribon \& Tavares Filho (2002), isto é, para se ter acurácia na estimativa da média em sistemas de preparo convencional, $\mathrm{n} \geq 25$.

No entanto, na grande parte dos trabalhos que usam penetrômetro de impacto para avaliar resistência do solo (Tormena \& Roloff, 1996; Stone \& Silveira, 1999; Tavares Filho et al., 1999a, 2001; Stone \& Moreira, 2001; Souza \& Alves, 2003; Teixeira et al., 2003; Barbosa et al., 2007) é comum o uso de três, cinco ou 10 repetições. Além disso, essa população amostral (três, cinco e 10), embora apresente maior intervalo de confiança, não apresenta diferença, a $5 \%$, entre os valores médios da maior população amostral $(n \geq 15)$. Considerando o trabalho de Davis et al. (1995), que utilizaram a percentagem do erro provável (D) de $10 \%$ (considerando esse erro aceitável em trabalhos dessa natureza) para o cálculo do número de amostras no estudo da variabilidade de propriedades químicas dos solos, estabeleceu-se a relação entre o tamanho das amostras e a acurácia da estimativa das médias de resistência do solo à penetração (Figura 4). Pode-se observar que o erro diminui rapidamente à medida que aumenta o número de amostras, em torno de 15 amostras nos manejos PD e CP e em torno de 20 no manejo PC, a partir do qual se estabiliza para uma acurácia da estimativa da média de $10 \%$, concordando com o trabalho de Davis et al. (1995).

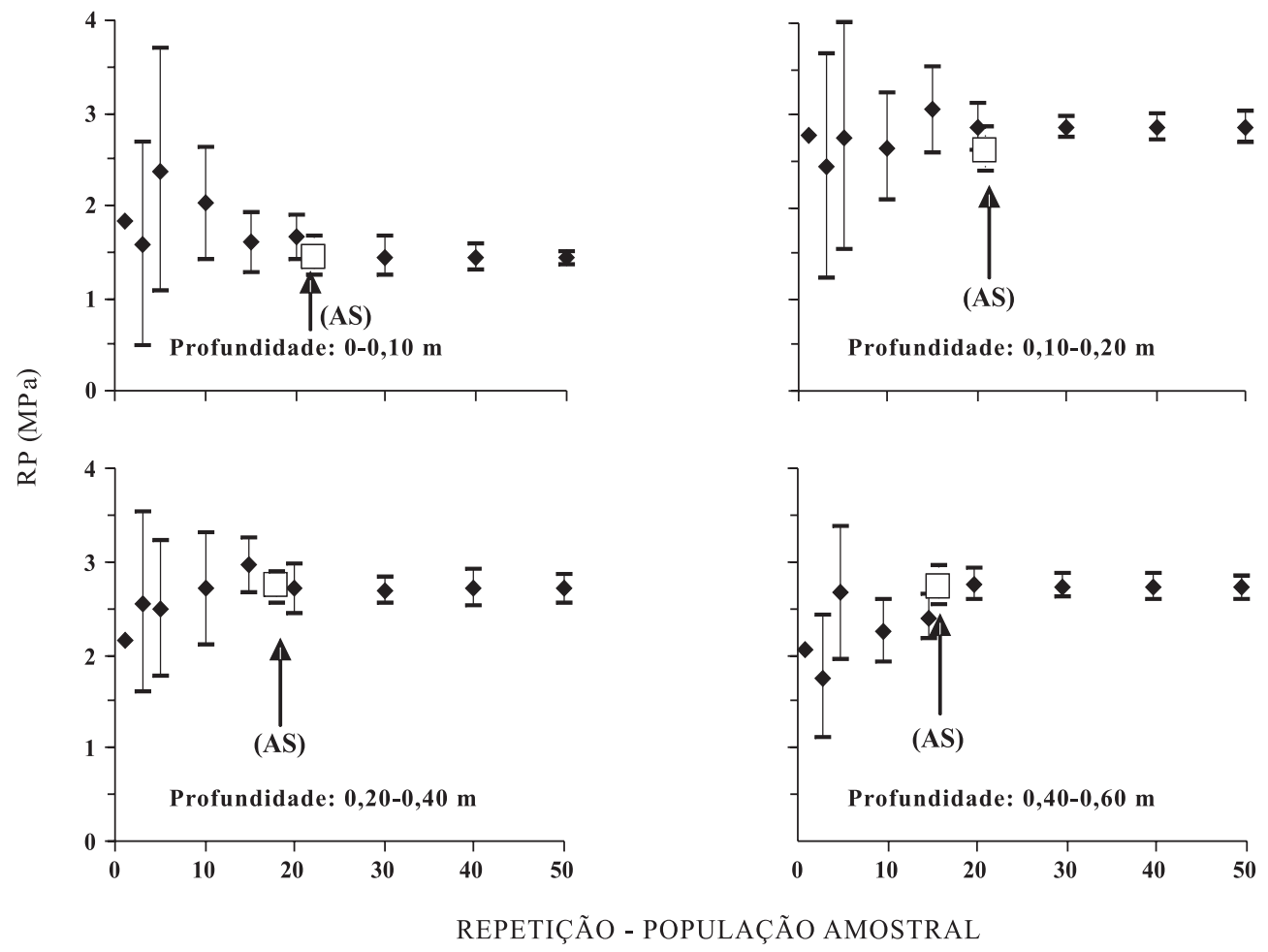

Figura 3. Valores médios e intervalo de confiança (95 \%) da resistência do solo à penetração (RP) função da amostragem ao acaso (clássica) e da amostragem sistemática (AS), com penetrômetro de impacto em diferentes profundidades de um Latossolo Vermelho eutroférrico sob preparo convencional. 
(a) Plantio direto (PD)

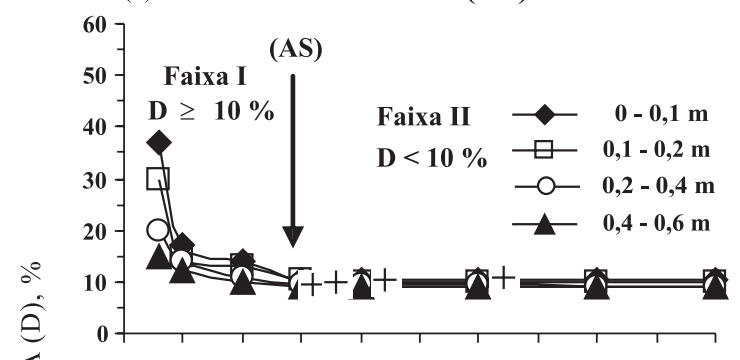

(b) Cultura perene (CP)

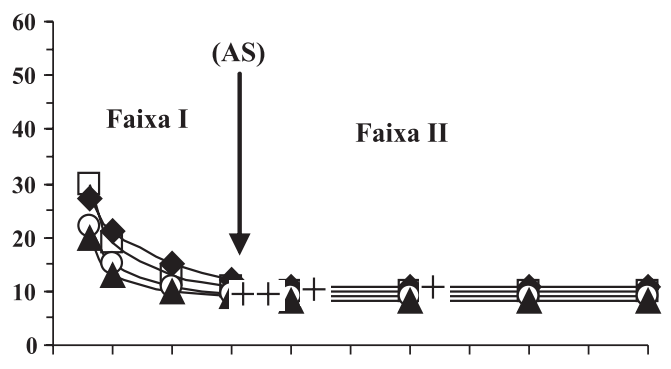

(c) Preparo convencional (PC)

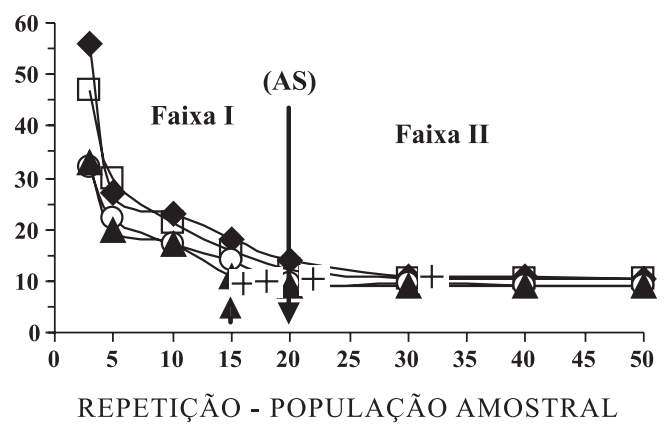

Figura 4. Acurácia da estimativa das médias de resistência do solo à penetração de um Latossolo Vermelho eutroférrico sob plantio direto (a), cultura perene (b) e sob preparo convencional (c), em função da amostragem ao acaso e da amostragem sistemática $(\mathrm{AS} ;+)$.

Dessa forma, as curvas apresentadas na figura 4 podem ser "divididas em duas faixas": faixa I, com $\mathrm{D} \geq 10 \%$ e faixa II, com $\mathrm{D}<10 \%$ determinadas pelo ponto de curvatura máxima, que se estabiliza a partir do índice de variabilidade (no caso, $\mathrm{D}=10 \%$ ). $\mathrm{Na}$ faixa I ( $\geq 10 \%$ ), em que ocorre maior variação dos resultados com maior variabilidade nos valores de resistência do solo à penetração e compreende a população amostral (n) $\leq 10$ para a profundidade 0-0,60 m, nos dois tipos de amostragem e manejos de solos estudados, deve-se tomar mais cuidado para se chegar a uma conclusão, por exemplo com relação à compactação do solo e restrição ao crescimento radicular de culturas. Nesse caso, um diagnóstico qualitativo (distribuição espacial das estruturas no perfil do solo), por exemplo por meio do estudo do perfil cultural (Tavares Filho et al., 1999b) e quantitativo (grau da compactação do solo a partir da resistência à penetração) ganha importância para auxiliar na verificação da qualidade do manejo utilizado e também no estabelecimento de limites de compactação que não afetem o crescimento radicular das plantas nos diferentes sistemas de manejo, como demonstrado por Tavares Filho et al. (2001).

$\mathrm{Na}$ faixa II ( $\mathrm{D}<10 \%)$, em que ocorre a melhor representatividade da média dos resultados de resistência do solo à penetração, compreendendo uma população amostral mínima (n) maior que 10 amostras nos dois tipos de amostragem e manejos de solos estudados, há maior confiabilidade nas médias obtidas na avaliação qualitativa (grau de compactação do solo a partir da resistência à penetração) do manejo utilizado e de estabelecimento de limites de compactação que não afetem o crescimento radicular das plantas nos diferentes sistemas de manejo, pois há alta acurácia dos dados, o que torna, os parâmetros estatísticos mais confiáveis, uma vez que ocorre maior homogeneidade nos resultados, evidenciada pela linearidade, das curvas das populações amostrais a partir do erro amostral de $10 \%$.

\section{CONCLUSÕES}

1. O número de amostras representativas para a determinação da resistência do solo à penetração não variou de forma significativa com o tipo de amostragem. Porém, os intervalos de confiança foram sempre menores na amostragem sistemática.

2. O número de amostras representativas para a determinação da resistência do solo à penetração variou com o sistema de manejo e a profundidade de amostragem. A melhor representatividade da média dos resultados de resistência do solo à penetração ocorreu para $n \geq 15$ (PD e CP) ou $n \geq 20(0-0,10 \mathrm{~m})$ e $15(0,20-0,60 \mathrm{~m})$ no caso do PC.

3. A população amostral $n \geq 10$ para a profundidade 0-0,60 m, nos dois tipos de amostragem e manejos de solos estudados, permitiu alta acurácia dos dados, tornando os parâmetros estatísticos mais confiáveis, com maior homogeneidade nos resultados e linearidade apresentada nas curvas das populações amostrais a partir do erro amostral de $10 \%$.

\section{LITERATURA CITADA}

BARBOSA, G.M.C.; TAVARES FILHO, J. \& FONSECA, I.C.B. Efeito do lodo de esgoto em propriedades físicas de um Latossolo Vermelho eutroférrico. Semina-Ciências Agr., 28:65-70, 2007.

BEAR, J. Dynamics of fluids in porous media. New York, Elsevier, 1972. 764p. 
BUCHTER, B.; HINZ, C. \& FLUHLER, H. Sample size for determination of coarse fragment content in a stony soil. Geoderma, 63:265-275, 1994.

CAMARGO, O.A \& ALLEONI, L.R.F. Compactação do solo e desenvolvimento de plantas. Piracicaba, Escola Superior de Agricultura Luiz de Queiroz, 1997. 132p.

CLAY, D.E.; CARLSON, C.G.; CHANG, J.; CLAY, S.A. \& MALO, D.D. Systematic evaluation of precision farming soil sampling requirements. In: ROBERT, P.C.; RUST, R.H. \& LARSON, W.E., eds. INTERNATIONAL CONFERENCE ON PRECISION AGRICULTURE, 4., Madison, 1999. Proceedings. Madison, ASA-CSSA-SSSA, 1999. p.253-265.

DAVIS, J.G.; HOSSNER, L.R.; WILDING, L. \& MANU, A. Varibility of soil chemical properties in two sandy, dunal soils of Niger. Soil Sci., 159:321-330, 1995.

EMBRAPA AGROPECUÁRIA OESTE. Recomendações da Comissão Centro Sul Brasileira de Pesquisa de Trigo para Mato Grosso do Sul. Dourados, 2002. 79p.

IMHOFF, S.; PIRES DA SILVA, A. \& TORMENA, C.A. Aplicações da curva de resistência no controle da qualidade física de um solo sob pastagem. Pesq. Agropec. Bras., $35: 1493-1500,2000$

JOBIM, C.C. Estabelecimento de pastagens. In:RAUEN, M.J.; SÁ, J.C.M. \& OLIVEIRA, E.F. Forragicultura no Paraná Londrina, Universidade Estadual de Londrina, 1996. p.3747.

LAUREN, J.G.; WAGENET, R.J.; BOUMA, J. \& WOSTEN, J.H.M. Variability of satured hydraulic condutivity in a Glossaquic hapludalf with macropores. Soil Sci., 145:2028, 1988.

MALLANTS, D.; MOHANTY, R.P.; VERVOOT, A. \& FEYEN, J. Spatial analysis of satured hydraulic condutivity in a soil with macropores. Soil Technol., 10:115-131, 1997.

PETERSEN, R.G. \& CALVIN, L.D. Sampling. In: BLACK, C.A., ed. Methods of soil analysis: Physical and mineralogical properties, including statistics of measurement and sampling. Madison, American Society of Agronomy, 1965. Part 1. p.54-71.

RIBON, A.A. \& TAVARES FILHO, J. Model for the estimation of physical quality of a Yellow Red Latosol (Oxisol) under pasture. Braz. Arch. Biol. Technol., 47:25-31, 2004.

RIBON, A.A. \& TAVARES FILHO, J. Número ideal de amostras para estudos com penetrômetros de impacto em Latossolo Vermelho eutroférrico. In: REUNIÃO BRASILEIRA DE MANEJO E CONSERVAÇÃO DO SOLO E DA ÁGUA, 14., Cuiabá, 2002. Resumos. Cuiabá, 2002.
SILVA, A.P.; LIBARDI, P.L. \& VIEIRA, S.R. Variabilidade espacial da resistência à penetração de um Latossolo Vermelho-Escuro ao longo de uma transeção. R. Bras. Ci. Solo, 13:1-5, 1989.

SOUZA, Z.M. \& ALVES, C. Movimento de água e resistência à penetração em um Latossolo Vermelho distrófico de cerrado, sob diferentes usos e manejos. R. Bras. Eng. Agríc. Amb., 7:18-23, 2003.

STOLF, R. Teoria e teste experimental de fórmulas de transformação dos dados de penetrômetro de impacto em resistência do solo. R. Bras. Ci. Solo, 15:249-252, 1991.

STOLF, R.; FERNANDES, J. \& FURLANI NETO, V.L. Penetrômetro de impacto IAA/PLANALSUCAR-STOLF; recomendação para seu uso. STAB, Açúcar Alcool Subpr., $1: 18-23,1983$

STONE, L.F. \& MOREIRA, J.A.A. Resposta do feijoeiro ao nitrogênio em cobertura, sob diferentes lâminas de irrigação e preparos do solo. Pesq. Agropec. Bras., 36:473$481,2001$.

STONE, L.F. \& SILVEIRA, P.M. Efeitos do sistema de preparo na compactação do solo, disponibilidade hídrica e comportamento do feijoeiro. Pesq. Agropec. Bras., 34:8391, 1999.

TAVARES FILHO, J.; BARBOSA, G.M.C.; GUIMARÃES, M.F. \& FONSECA, I.C.B. Resistência do solo á penetração e desenvolvimento do sistema radicular do milho (Zea mays) sob diferentes sistemas de manejo em um Latossolo Roxo. R. Bras. Ci. Solo, 25:725-730, 2001.

TAVARES FILHO, J.; EIRA, G.C. \& FARINHA, L.R.L. Avaliação da compactação do solo em um solo cultivado no sistema convencional. Eng. Agríc., 19:219-225, 1999a.

TAVARES FILHO, J.; RALISCH, R.; GUIMARÃES, M.F.; MEDINA, C.C.; BALBINO, L.C. \& NEVES, C.S.V.J. Método do perfil cultural para avaliação do estado físico de solos em condições tropicais. R. Bras. Ci. Solo, 23:39399, 1999b.

TEIXEIRA, C.F.A.; PAULETTO, E.A. \& SILVA, J.B. Resistência mecânica à penetração de um Argissolo Amarelo distrófico típico sob diferentes sistemas de produção em plantio direto. Ci. Rural, 33:1165-1167, 2003.

TORMENA, C.A.; SILVA, A.P. \& LIBARDI, P.L. Caracterização do intervalo hídrico ótimo de um Latossolo Roxo sob plantio direto. R. Bras. Ci. Solo, 22:573-581, 1998.

TORMENA, C.A. \& ROLOFF, G. Dinâmica da resistência à penetração de um solo sob plantio direto. R. Bras. Ci. Solo, 20:333-39, 1996.

TORRES, E. \& SARAIVA, O.F. Camadas de impedimento do solo em sistemas agrícolas com a soja. Londrina, Embrapa Soja, 1999. 58p. (Circular Técnica, 23) 